\title{
Dissociable Neural Responses Related to Pain Intensity, Stimulus Intensity, and Stimulus Awareness within the Anterior Cingulate Cortex: A Parametric Single-Trial Laser Functional Magnetic Resonance Imaging Study
}

\author{
Christian Büchel, ${ }^{1}$ Karin Bornhövd, ${ }^{1}$ Markus Quante, ${ }^{2}$ Volkmar Glauche, ${ }^{1}$ Burkhard Bromm, ${ }^{2}$ and \\ Cornelius Weiller ${ }^{1}$ \\ ${ }^{1}$ Cognitive Neuroscience Laboratory, Department of Neurology, and 2Department of Physiology, Hamburg University \\ Medical School, Hamburg, D-20246 Germany
}

Neuroimaging studies have demonstrated activations in the anterior cingulate cortex (ACC) related to the affective component of pain, but not to stimulus intensity. However, it is possible that the low spatial resolution of positron emission tomography, as used in the majority of these studies, obscured areas coding stimulus intensity. We revisited this issue, using a parametric single-trial functional magnetic resonance imaging design, and investigated pain, stimulus intensity, and stimulus awareness (i.e., pain unrelated) responses within the ACC in nine healthy volunteers. Four different stimulus intensities ranging from warm to painful (300-600 $\mathrm{mJ}$ ) were applied with a thulium yttrium-aluminum granate infrared laser in a randomized order and rated by the subjects on a five point scale (P0-P4).

Pain-related regions in the ventral posterior ACC showed a response that did not distinguish between innocuous trials (P0 and $\mathrm{P} 1$ ) but showed a positive linear relationship with the blood oxygenation level-dependent contrast signal for painful trials (P2-P4). Regions in the dorsal anterior ACC along the cingulate sulcus differentiated between P0 (not perceived) and P1 but exhibited no additional signal increase with P2; these regions are related to stimulus awareness and probably to cognitive processing. Most importantly, we identified a region in the dorsal posterior ACC showing a response that discriminated between nonpainful trials ( $\mathrm{P0}$ and $\mathrm{P} 1$ ); therefor, this region was simply related to basic sensory processing and not to pain intensity. Stimulus-related activations were all located adjacent to the cingulate motor area, highlighting the strategic link of stimulus processing and response generation in the posterior ACC.

Key words: pain; single-trial fMRl; laser; neuroimaging; parametric; stimulus response function; affect; emotion; sensory coding; cingulate motor area; anterior cingulate cortex
The anterior cingulate cortex (ACC) plays an important role in pain processing (Devinsky et al., 1995). Its pivotal role within the nociceptive system has been established in lesion studies (Foltz and White, 1968; Bouckoms, 1989; Talbot et al., 1995) and in numerous functional neuroimaging studies using positron emission tomography (PET) (Jones et al., 1991; Talbot et al., 1991; Casey et al., 1994; Craig et al., 1996; Rainville et al., 1997; Coghill et al., 1999; Casey, 2000) and functional magnetic resonance imaging (fMRI) (Davis et al., 1997; Porro et al., 1998; Ploghaus et al., 1999; Kwan et al., 2000; Sawamoto et al., 2000). The ACC receives nociceptive inputs via medial thalamic nuclei (Vogt and Pandya, 1987; Vogt et al., 1987) and has been shown to code affective components of painful stimuli (Craig et al., 1996; Rainville et al., 1997; Sawamoto et al., 2000). It is involved in working memory (Petit et al., 1998) and attentional processing (Gitelman et al., 1999; Peyron et al., 1999), and therefore is well suited to shift attention toward pain. It is also involved in learning associations between aversive and neutral stimuli in classical condition-

\footnotetext{
Received Sept. 4, 2001; revised Oct. 24, 2001; accepted Oct. 25, 2001.

This work was supported by the Volkswagenstiftung. We thank the Physics and Methods group at the Cognitive Neuroscience Laboratory in Hamburg, the technical staff at the Department of Neurophysiology for their support with the laser, and J. Lorenz, D. Gonzalo, A. Schnitzler, and U. Bingel for suggestions on a previous draft of this paper.

Correspondence should be addressed to Dr. Christian Büchel, Neurologische Universitätsklinik, Haus B, Universitäts-Krankenhaus Eppendorf, Martinistrasse 52, D-20246 Hamburg, Germany. E-mail: buechel@uke.uni-hamburg.de. Copyright (C) 2002 Society for Neuroscience $0270-6474 / 02 / 220970-07 \$ 15.00 / 0$
}

ing (LaBar et al., 1998; Ploghaus et al., 1999; Büchel and Dolan, 2000), which is important to avoid future damage in a similar situation, and it embodies higher motor areas [cingulate motor area (CMA)] (Dum and Strick, 1996; Picard and Strick, 1996; Fink et al., 1997), which are necessary for defense preparation.

Most studies, including those manipulating pain affect with hypnosis (Rainville et al., 1997) or those investigating an illusion leading to pain (Craig et al., 1996), have only found evidence for affective or pain-related processing within the ACC. So far, simple sensory coding of stimulus intensity in the ACC has not been observed, and in a recent review, Peyron et al. (2000) had to conclude that there is no evidence "favoring a role of ACC in coding stimulus intensity."

It should be noted that only few previous imaging studies allowed the identification of stimulus intensity-related responses, because they used at least two stimulus intensities below pain threshold (Casey et al., 1996; Tölle et al., 1999). This concept was taken further by a parametric PET study that allowed assessment of the relationship between brain activity and pain intensity (Coghill et al., 1999). In this study, linear regression analysis revealed pain intensity-related activation in the ACC. All of these studies used PET; therefore a response profile indicative of sensory processing in a small region might have been obscured by neighboring pain-related activations.

We revisited this issue with event-related fMRI in combination with a thulium yttrium-aluminum granate (YAG) infrared laser 
that delivers very brief heat stimuli, in a parametric design, with four different stimulus intensities ranging from warm to painful. This allowed us to assess individual blood oxygenation leveldependent (BOLD) responses as a function of stimulus and pain intensity and to plot a stimulus response function (SRF) for each region similar to the study by Coghill et al. (1999).

We focused on two distinct shapes of this function: (1) an immediate linear increase discriminating between low, innocuous intensities, indicative of sensory-discriminative processing, and (2) an initial plateau followed by a linear increase only at higher, painful intensities (i.e., a step function with no discrimination of nonpainful warm stimuli and an increase for painful stimuli, indicative of pain processing).

\section{MATERIALS AND METHODS}

Subjects. A total of 10 healthy subjects ( 7 male, 3 female) with a mean age of 28.1 years (range 24-42 years) were recruited from the University of Hamburg; subjects gave their written informed consent to participate in the study, which was approved by the local Ethics Committee. One subject had to be excluded from the study because of an exceedingly high pain threshold and poor pain-discrimination ability. The remaining nine subjects (six male, three female; one left-handed) were free to withdraw from the study at any time.

During scanning, two investigators stayed with the subject in the scanner room. One investigator applied the laser stimuli to the dorsum of the left hand and the other investigator documented the rating of each stimulus.

Laser stimulator. A thulium YAG laser (Baasel Lasertech, Starnberg, Germany) was used to apply computer-controlled brief radiant pulses to the skin of the subjects. The thulium laser emits near-infrared radiation (wavelength of $1.96 \mu \mathrm{m}$, spot diameter of $5 \mathrm{~mm}$, pulse duration of $1 \mathrm{msec}$ ) with a penetration depth of $360 \mu \mathrm{m}$ into human skin. The laser stimulus allows a precise restriction of the emitted heat energy to the termination area of primary nociceptive afferents $(20-570 \mu \mathrm{m})$ without damaging the epidermis or affecting the subcutaneous tissue (Spiegel et al., 2000). In addition, the temperature rise in the superficial skin after laser stimuli is fast enough to elicit activations of thinly myelinated $\mathrm{A} \delta$ nociceptors and unmyelinated $\mathrm{C}$ nociceptors.

Experimental protocol and pain rating. In a single fMRI session, 100 nociceptive stimuli were delivered to the dorsum of the left hand. Interstimulus intervals were randomized in an interval between 8 and 12 sec to minimize pain anticipation (Bromm and Treede, 1991). The stimulation site was changed slightly after each stimulus to avoid nociceptor sensitization and fatigue. Stimulation intensity was randomized between 300 and $600 \mathrm{~mJ}$ ( 300 to 400 to 500 to $600 \mathrm{~mJ}$ ). At $4 \mathrm{sec}$ after the laser stimulus, a tone $(1 \mathrm{kHz}$ sine, $500 \mathrm{msec})$ signaled the subjects to rate the perceived pain intensity by giving signs with their right hand. Showing a closed hand indicated a pain intensity rating of zero (P0); one finger (thumb) indicated a pain intensity of one (P1); two, three, or four fingers indicated a pain intensity of $\mathrm{P} 2, \mathrm{P} 3$, and $\mathrm{P} 4$, respectively. The intensities were defined as follows: P0, no stimulus perceived; P1, a clear, warm but not painful sensation; P2, P3, and P4, increasingly painful sensations, comparable to a pinprick. P4 was defined as the most intensive pain stimulus of the study. Volunteers were exposed to all pain intensities and trained with this rating scale inside the MR scanner for 20 min before scanning. In addition, the individual pain threshold was derived psychophysically in each subject before scanning by three series of stimuli ascending in steps of $30 \mathrm{~mJ}$, from below sensation threshold to $90 \mathrm{~mJ}$ above pain threshold, and back again to below sensation threshold. Data pertaining to this prescanning pain threshold estimation were lost for two subjects, because of a computer failure. Because of the low intersubject variability of the individual pain threshold in previous behavioral studies, we decided to use fixed energy levels for all subjects to simplify the paradigm and the data analysis (Bromm and Lorenz, 1998).

Image acquisition. MR scanning was performed on a $1.5 \mathrm{~T}$ MR scanner (Siemens Vision, Erlangen, Germany). In a single session, 375 volumes ( 25 contiguous, axial, 3 -mm-thick slices each; $1 \mathrm{~mm}$ gap) were acquired using a gradient echo echo-planar imaging (EPI) T2*-sensitive sequence (repetition time, $2800 \mathrm{msec}$; echo time, $60 \mathrm{msec}$; flip angle, $90^{\circ}$; matrix, $64 \times 64$; field of view, $210 \times 210 \mathrm{~mm}$ ). A standard head coil was used and packed with foam pads. Subjects were blindfolded during the experiment. For display purposes, a high-resolution $(1 \times 1 \times 1 \mathrm{~mm}$ voxel size $)$

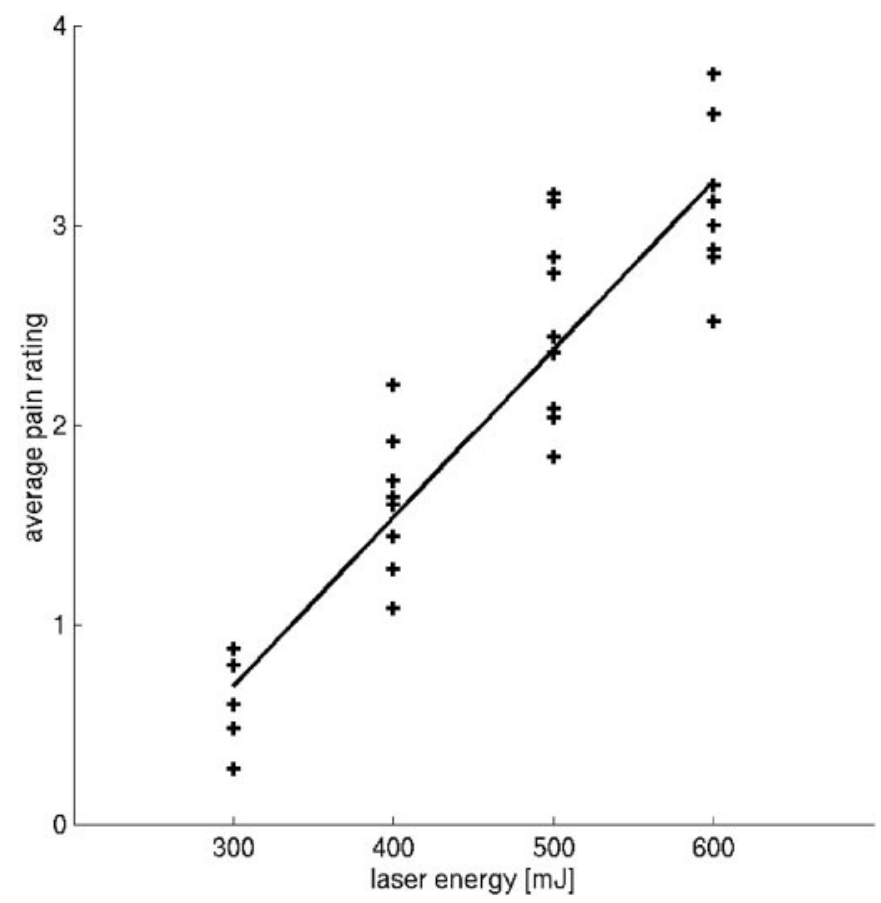

Figure 1. Relationship between applied stimulus energy ( $x$-axis) and average rating ( $\mathrm{P} 0-\mathrm{P} 4 ; y$-axis). Each plotted data point refers to the mean rating for each energy level and each subject (i.e., nine data points for each energy level). The average pain rating was linearly related to laser energy (average pain rating $=$ laser energy $\times 0.84-0.15$ ), where laser energy is $300,400,500$, and $600 \mathrm{~mJ}$.

$\mathrm{T}_{1}$-weighted structural MRI was acquired for each volunteer using a three-dimensional fast low angle shot sequence.

Image processing and statistical analysis. Image processing and statistical analysis were performed using SPM99 (Friston et al., 1995b; Worsley and Friston, 1995) (http://www.fil.ion.ucl.ac.uk/spm). All volumes were realigned to the first volume (Friston et al., 1995c), spatially normalized (Friston et al., 1995a) to a standard EPI template (Evans et al., 1993), and finally smoothed using a $6 \mathrm{~mm}$ full width at half maximum isotropic Gaussian kernel. Data analysis was performed by modeling the different trials ("perceived pain intensity P0, P1, P2, P3, P4") as $\delta$ functions convolved with a set of two basis functions, modeling an early response peaking at $5 \mathrm{sec}$ after application of the painful stimulus and a second basis function peaking at $9 \mathrm{sec}$ (expected peak for the motor component). The basis functions were the canonical hemodynamic response function (HRF) as implemented in SPM99. Voxelwise regression coefficients for all regressors were estimated using least squares within SPM99 (Friston et al., 1995c).

Specific effects were tested with appropriate linear contrasts of the regression coefficients (parameter estimates), resulting in a $t$ statistic for each voxel. These $t$ statistics constitute a statistical parametric map (SPM). SPMs are interpreted by referring to the probabilistic behavior of Gaussian random fields (Worsley, 1994). Data were analyzed for each subject individually and with a fixed-effects model to make inferences about the entire group of subjects (Friston et al., 1999).

Because of strong a priori hypotheses of pain-related responses in the ACC, the threshold was set to $p<0.001$ (uncorrected). The $\mathrm{T}_{1}$-weighted structural volume was coregistered to the functional scans by normalizing it to a $T_{1}$-weighted template in the same space as the $T_{2}{ }^{*}$ EPI template used to normalize the functional data set.

\section{RESULTS}

\section{Behavioral data}

The correlation coefficient between perceived intensity and applied stimulus intensity were $0.72,0.67,0.81,0.72,0.70,0.82,0.80$, 0.69 , and 0.75 in individual subjects. Figure 1 shows the relationship between average pain rating and applied stimulus intensity. 
The average pain rating was linearly related to laser energy (average pain rating $=$ laser energy $\times 0.84-0.15$ ), where laser energy is $300,400,500$, and $600 \mathrm{~mJ}$.

On average, a $\mathrm{P} 0$ rating was associated with a mean intensity of $333.5 \pm 5.7 \mathrm{~mJ}$; P1 was associated with a mean intensity of $375.6 \pm 11.3 \mathrm{~mJ}, \mathrm{P} 2$ was associated with a mean intensity of $446.0 \pm 9.5 \mathrm{~mJ}, \mathrm{P} 3$ was associated with a mean intensity of $527.2 \pm 7.5 \mathrm{~mJ}$, and P4was associated with a mean intensity of $577.4 \pm 5.3 \mathrm{~mJ}$. Perceived intensity was linearly related to laser energy (applied energy $=$ pain rating $\times 63.9+324$ ), where rating is from 0 to 4 . The pain threshold during MRI scanning (average of mean intensity associated with P1 and P2) was $410 \pm 28 \mathrm{~mJ}$.

\section{fMRI data}

The main aim of the study was the characterization of areas activated by painful stimuli by their relationship to stimulus intensity and pain in the ACC (Büchel et al., 1998). Activations outside the ACC are beyond the scope of this paper and will be reported elsewhere. Treating differently rated trials as different conditions allowed us to analyze the relationship between stimulus properties (pain and intensity) and BOLD signal.

Fitting canonical HRFs (see Materials and Methods for details) to the data yields a regression coefficient indicating the magnitude of the response for each trial type (P0-P4). This magnitude of the regression coefficient plotted as a function of rating is the SRF (see Fig. 3, middle column).

The rating scale used is linear with respect to stimulus intensity as defined by the energy level of the laser (Fig. 1). However, the scale is deliberately nonlinear with respect to pain. P0 and P1 were both defined as nonpainful, whereas P2-P4 were linearly related to increasing pain intensity.

Essentially, we found three different SRFs: (1) Some areas showed a significantly higher BOLD signal for P1 compared with P0 but no additional signal increase with P2-P4. Given that P0 was used to code stimuli that were not perceived, this simple step function discriminates between perceived and nonperceived stimuli without any additional pain or intensity discrimination and is related to stimulus perception (i.e., attentional and working memory processing) (Fig. 2a). (2) Some areas showed a linear relationship beginning at $\mathrm{P} 0$ (i.e., distinguished well between $\mathrm{P} 0$, $\mathrm{P} 1$, and P2). This SRF differentiates between different stimulus intensities, although some (P0 and P1) were perceived as nonpainful. Signal changes in these regions are therefore related to stimulus intensity (Fig. 2b). (3) Other SRFs showed an initial plateau (i.e., did not differentiate between P0, P1, and sometimes P2) but showed a linear relationship for P2-P4. Given the definition of our pain-rating scale, in which $\mathrm{P} 2$ was defined as clearly painful and P0 and P1 were defined as nonpainful, this type of SRF is indicative of areas coding pain intensity (Fig. 2c).

\section{Areas showing a stimulus perception-related SRF}

Regions with such an SRF were identified by a contrast comparing P1 with P0. To further exclude regions that also show differences between $\mathrm{P} 2$ and $\mathrm{P} 1$, this contrast was exclusively masked with the contrast $\mathrm{P} 2>\mathrm{P} 1$ at $p<0.001$. In simple words, this masked contrast reveals areas that show a significant difference

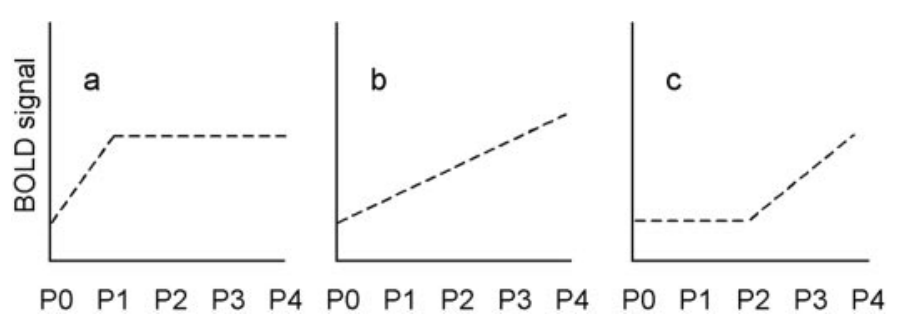

Figure 2. Different SRFs. The SRF in $a$ shows a higher BOLD signal for P1 (stimulus perceived, but not painful) compared with P0 (stimulus not perceived), but no additional signal increase with P2-P4 (low level, mid-level, and high level of pain). This step function discriminates between perceived and unperceived stimuli without any additional pain or intensity discrimination and seems to be linked to stimulus awareness and possibly to cognitive processing (e.g., working memory or attention). The SRF in $b$ shows a linear relationship beginning at $\mathrm{P} 0$ and differentiates well between stimulus intensities, although some (P0 and P1) were perceived as nonpainful. This SRF is related to stimulus intensity. The SRF depicted in $c$ shows an initial plateau (i.e., does not differentiate between P0 and P1 but shows a linear relationship for P2-P4). According to our pain-rating scale, in which $\mathrm{P} 2$ was defined as clearly painful and P0 and P1 were not painful, this SRF is related to pain intensity.

between $\mathrm{P} 1$ and $\mathrm{P} 0$ but not between $\mathrm{P} 1$ and $\mathrm{P} 2$. Areas showing such an SRF were found in an area in the dorsal anterior ACC (aACC) $(y=21-36 \mathrm{~mm})$ covering the cingulate sulcus (Fig. $3 a$, blue areas and middle plot). Table 1 summarizes the significance and location of these results. As indicated in Figure 3, we use the nomenclature of Kwan et al. (2000) to describe subregions in the ACC. They divide the ACC into the aACC and the posterior part (pACC).

\section{Areas showing a stimulus intensity-related SRF}

To identify regions showing intensity-related BOLD signal changes, we used a contrast modeling a linear signal increase for $\mathrm{P} 0-\mathrm{P} 3$. The most prominent linear relationship of the BOLD signal with stimulus intensity in the low stimulus range (P0-P2) was observed in the posterior part of the cingulate sulcus. Apart from a linear relationship at low stimulus intensities, this area showed a ceiling effect (i.e., a slight decrease rather than an increase in the BOLD signal) when moving from P3 to P4 (Fig. $3 a$, red areas and top plot).

\section{Areas showing a pain-related SRF}

To identify regions showing a pain-related BOLD signal, we used a contrast modeling a linear signal increase for P1-P4, without significant difference between P0 and P1. Areas showing a linear relationship between BOLD signal and pain were found in the perigenual ACC and in the pACC (Table 1) (Fig. 3a, green areas and bottom plot). Inspection of the spatial distribution of stimulus intensity- and pain-related response functions revealed that the peak of the pain-related activations was lateralized to the right (i.e., contralateral to the stimulated hand). A clear anteriorposterior distribution was observed for pain- and attentionrelated areas, with pain-related areas covering the pACC (i.e., posterior to the attention-related aACC activations). The stimulus intensity-related areas were found dorsally to the pain-related

(Figure legend continued.) The middle column shows the individual SRF for each region. Fitting canonical HRFs (see Materials and Methods for details) to the data yields a regression coefficient indicating the magnitude of the response for each trial type (P0-P4). This magnitude ( \pm SEM) plotted as a function of rating is the SRF. In the right column, adjusted data in each region are plotted in bins of 2 sec as a function of peristimulus time separately for $\mathrm{P} 0-\mathrm{P} 4$. In $b$, the motor-evoked hemodynamic response in the CMA is plotted. Additional motor-evoked responses were observed in the SMA. 

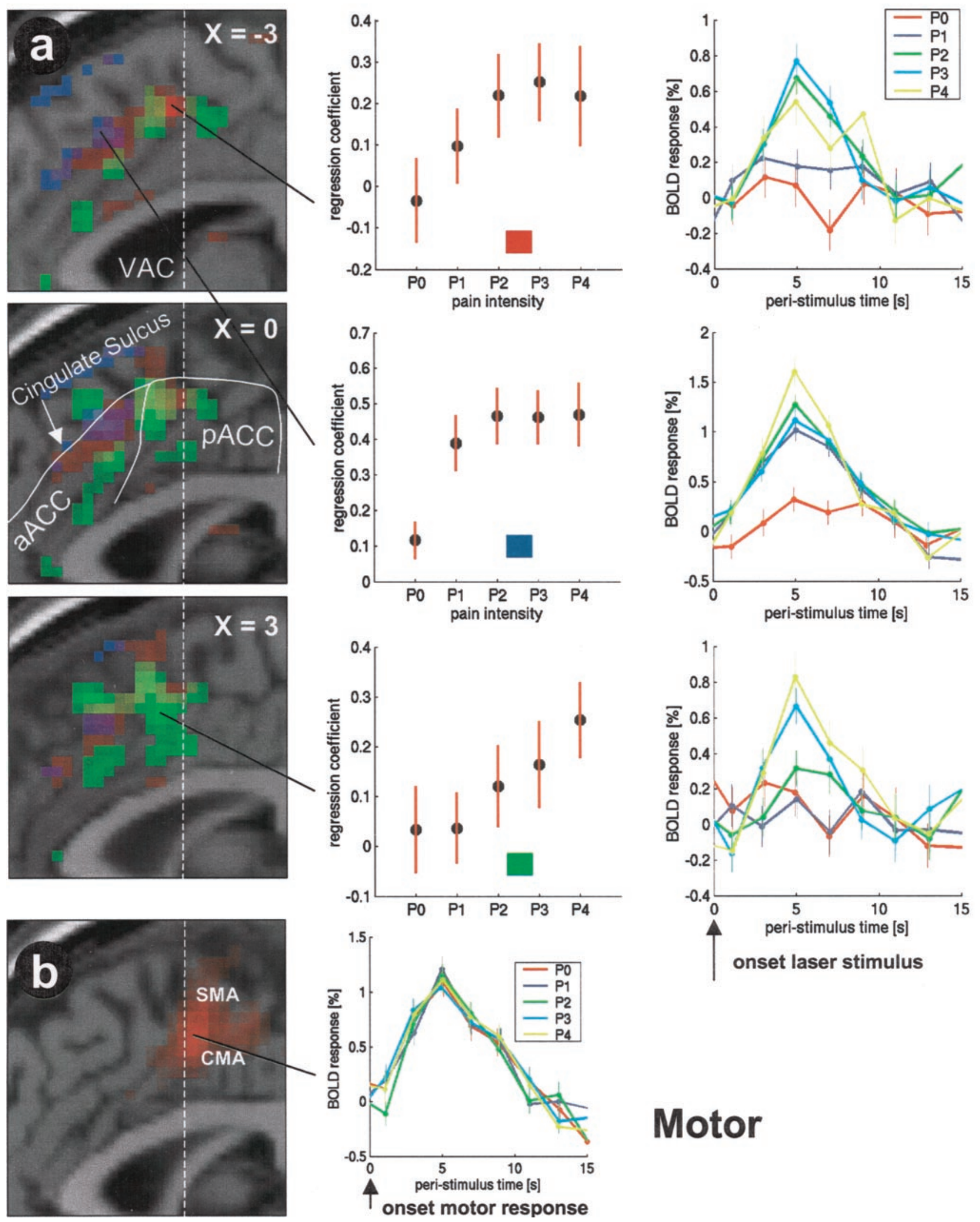

\section{Motor}

Figure 3. Activations in the ACC $(p<0.001)$ overlaid on a structural $\mathrm{T}_{1}$-weighted MRI used for spatial normalization. Laser-evoked responses and their locations are depicted in $a$. Three sagittal slices through the ACC are shown from $\mathrm{x}=-3 \mathrm{~mm}$ to $\mathrm{x}=3 \mathrm{~mm}$. The dashed white line indicates the vertical line through the anterior commissure $(V A C)$. Regions showing different SRFs are color-coded. Stimulus intensity-related areas are shown in red, pain intensity-related areas are shown in green, and cognitive processing-related areas are shown in blue. The top row shows an area in the dorsal pACC with a stimulus intensity-related (red) SRF. The middle row shows the cognitive processing-related step function response of a region in the cingulate sulcus of the aACC (blue). The bottom row shows the SRF of a pain intensity-related area in the ventral pACC (green). (Figure legend continues.) 


\begin{tabular}{llll}
\hline \multicolumn{3}{l}{ Table 1. Activations in the anterior cingulate cortex } & \\
Stimulus response type & Region & $x, y, z(\mathrm{~mm})$ & $Z$ \\
\hline Stimulus perception, at & aACC & $-3,21,45$ & $4.6^{*}$ \\
tention, and working & & $-3,36,33$ & $4.1^{*}$ \\
memory & & & \\
Stimulus intensity- & Dorsal pACC & $-3,3,51$ & $6.9^{* *}$ \\
related & & $-3,18,42$ & $5.8^{* *}$ \\
Pain-related & Ventral pACC & $3,6,48$ & $4.8^{*}$ \\
& & $3,6,36$ & $3.8^{*}$ \\
& & $3,9,54$ & $5.1^{* *}$ \\
& & $0,18,36$ & $4.5^{* *}$ \\
& & $3,27,51$ & $5.5^{* *}$ \\
& Medial frontal cortex & $3,24,30$ & $3.8^{*}$ \\
& & $-6,54,12$ & $5.1^{* *}$ \\
& & $-6,36,-12$ & $4.3^{*}$ \\
\hline
\end{tabular}

${ }^{*} p<0.001 ;{ }^{*} p<0.05$, corrected.

activations in the pACC, again posterior to the unspecific activations in the cingulate sulcus of the aAC (Fig. $3 a$ ).

To test for differences in shape, a second-order polynomial $\left(y=b_{1} x^{2}+b_{2} x+b_{3}\right)$ was fitted to the SRF and the second-order coefficients $\left(b_{1}\right)$ were compared using a paired $t$ test. The painrelated SRF ( $x=3, y=6, z=48$ ) was significantly different from the stimulus intensity-related SRF $\left(x=-3, y=3, z=51 ; t_{(8)}=\right.$ $1.9 ; p<0.05)$ and the stimulus perception-related SRF $(x=-3$, $\left.y=21, z=45 ; t_{(8)}=2.4 ; p<0.05\right)$.

\section{Motor responses}

To identify regions that show motor-related activation, we contrasted the basis functions coding pain responses with those coding motor responses pooled over all stimulus intensities. Apart from the left (contralateral to the moving hand) primary motor cortex, motor-related responses were observed in the dorsal pACC, posterior to the pain-related activations. With regard to previous motor studies, these activations enclose the supplementary motor area (SMA) and the CMA (Fink et al., 1997; Petit et al., 1998; Kwan et al., 2000) (Fig. 3b).

\section{DISCUSSION}

Using a laser pain stimulus in combination with a parametric event-related fMRI design, we have characterized regions in the ACC according to their SRF as (1) stimulus perception-related, (2) stimulus intensity-related, or (3) pain-related. The ACC showed all three response types: a BOLD signal in the perigenual ACC and ventral pACC was related to pain intensity, whereas aACC signal changes were unspecific and most likely related to working memory and attentional processes. More importantly, we have characterized a dorsal pACC region in the cingulate sulcus coding stimulus intensity. Motor-related activations were found immediately posterior to the pain- and stimulus-related ACC activations in the SMA and CMA.

\section{Pain stimulus}

The thulium YAG laser is the ideal stimulation device for eventrelated fMRI studies of pain. It delivers brief $(1 \mathrm{msec})$ stimuli with defined energy levels and only activates nociceptors and not the vibrotactile sensory system. In recent magnet encephalography studies, it was shown that vibrotactile stimuli evoke very short latency responses in the primary somatosensory cortex, whereas with the laser, the latencies of the evoked responses in the SI [and secondary somatosensory cortex (SII)] were almost three times longer, without the initial short-latency component, highlighting the ability of the laser to exclusively stimulate nociceptors (Ploner et al., 2000; Timmermann et al., 2001).

\section{Pain intensity-related responses}

We applied four different pain intensities (300, 400, 500, and 600 $\mathrm{mJ}$ ), which were rated for intensity on a five-point rating scale ranging from 0 to 4 . The BOLD response was correlated with individual ratings instead of the applied intensities, because this allowed us to accurately dissociate painful ( $\geq \mathrm{P} 2)$ from nonpainful $(\leq \mathrm{P} 1)$ events.

Activation of the ACC in pain studies has exclusively been linked with nociceptive or affective processing. This finding has been derived by experimentally dissociating pain affect from stimulus intensity using hypnosis (Rainville et al., 1997) or using a pain illusion (Craig et al., 1996). Other studies used multiple intensities (Coghill et al., 1999) and linear regression to find regions related to pain perception (Tölle et al., 1999), and still others investigated a very intense pain and the decay of this sensation over time (Porro et al., 1998). The location of the main activation related to pain processing in our study was found in a mid-cingulate region slightly anterior to the anterior commissure $(x=3, y=6, z=48)$. This is close to an activation correlated with pain intensity in a previous fMRI study where the peak was located ventrally ( $x=7, y=6, z=34$ ) (Porro et al., 1998). This location is also in accord with another recent fMRI study investigating pain processing in the ACC (Kwan et al., 2000). A single neuron study performed in awake humans showed an SRF that was similar to that found in our study (Hutchison et al., 1999). Neurons in the ventral pACC showed no increase in firing rate with an increase in temperature in the low (nonpainful) temperature range, but a marked increase was seen in the painful range $\left(46-50^{\circ} \mathrm{C}\right)$. The peak of our pain-related activation also coincides with previous PET studies in which a pain intensity- $(x=-0.1$, $y=11.5, z=35.8$ ) (Coghill et al., 1999) or pain threshold-related activation was found in the pACC $(x=6, y=3, z=43)$ (Tölle et al., 1999).

\section{Stimulus intensity-related responses}

Based on previous reports, we exclusively expected activations correlated with pain, because almost all studies have implicated the ACC in the affective coding of pain (Rainville et al., 1997; Sawamoto et al., 2000). However, our data clearly show that a region in the dorsal pACC within the cingulate sulcus also exhibits stimulus intensity-related BOLD responses that are not related to pain intensity, because BOLD responses in this region differentiated well between $\mathrm{P} 0$ and $\mathrm{P} 1$, which were both innocuous trials (Fig. 3, red areas). Although previous studies have concentrated on affective or pain-related responses in the ACC, this type of activation is not totally unexpected, considering that medial and intralaminar thalamic nuclei from which the ACC receives input (Vogt and Pandya, 1987; Vogt et al., 1987) have shown intensity coding (Bushnell and Duncan, 1989). ACC neurons coding stimulus intensity have been found previously in the anesthetized rabbit (Sikes and Vogt, 1992). Furthermore, clinical data from patients with anterior cingulotomy (Davis et al., 1994) or deafferented anterior cingulate (Talbot et al., 1995) have also shown a diminished sensory coding ability. The only other region showing a similar SRF in our study was the primary sensory cortex. Similar to the SRF observed there, stimulus intensityrelated responses in the ACC showed a ceiling effect, or even a 
decrease, when moving from P3 to P4, suggesting an additional, possibly antinociceptive, mechanism initiated at higher stimulus intensities.

We can only speculate as to why stimulus intensity-related responses have not been found in previous functional neuroimaging studies. One reason might be that subtle differences in SRFs are obscured by low spatial resolution in those PET studies that used two or more innocuous stimulus conditions (Casey et al., 1996; Coghill et al., 1999; Tölle et al., 1999) or used averaging over the entire ACC in a region of interest in fMRI (Sawamoto et al., 2000). Furthermore, in most studies, stimulus intensities have been chosen to detect pain but not stimulus intensity-related activations (Kwan et al., 2000).

\section{Pain-intensity-independent responses}

Aside from ACC regions showing a relationship with stimulus or pain intensity, our study revealed a region in the aACC, within the cingulate sulcus, that showed a significant BOLD signal difference between $\mathrm{P} 0$ and $\mathrm{P} 1$ but no additional discrimination between P1, P2, P3, and P4. Similar SRFs were observed in the dorsolateral prefrontal cortex and the intraparietal sulcus. This "on-off" response profile is related to whether a stimulus was perceived (a rating of $>\mathrm{P} 0$ ). Alternatively, this SRF could be related to coding of warmth, in accordance with the fact that primary afferent warm fibers code intensity of warmth up to pain threshold and then either a plateau or decrease in activity in the pain range.

Whenever a stimulus is consciously perceived, many cognitive processes will be initiated: (1) an exogenous shift of spatial attention to the stimulated site (Corbetta et al., 2000), (2) rating of intensity, and (3) keeping this rating in working memory for 4 sec as required in our task. This interpretation is in agreement with a recent fMRI study on working memory-related responses in the medial wall (Petit et al., 1998). Recent single-cell work in patients undergoing cingulotomy revealed attention-related activation in aACC neurons (Davis et al., 2000), anterior to pain responsive neurons (Hutchison et al., 1999). The location of these responses is in close agreement with the peak of our binary response found in the aACC $(x=-3, y=21, z=45)$. Recently, an elegant study directly investigated the effect of attention in the context of pain processing using a full factorial design. In precise spatial agreement with regions showing an on-off SRF according to our data, the main effect of attention (regardless of whether the stimulus was noxious or innocuous) activated an area in the aACC $(x=-2, y=22, z=40)$ (Peyron et al., 1999).

\section{Motor}

Our experiment explicitly comprised an abstract motor command with the contralateral hand, thus avoiding interference with a possible withdrawal reflex (Schouenborg et al., 1992). Contrasting laser-evoked with motor-evoked signal changes, we found a large area in the dorsal pACC located within the boundary of the CMA and the SMA (Devinsky et al., 1995; Dum and Strick, 1996; Fink et al., 1997) (Fig. 3b, red areas).

In agreement with a previous study (Kwan et al., 2000), ACC subregions that were only activated by the motor task were located in close vicinity to pain- and stimulus intensity-related activations (Fig. $3 b$ ). Such a spatial configuration (i.e., affective and sensory areas neighboring the response-generating motor areas) seems advantageous, because the generation of an adequate response requires as much information about the painful stimulus as possible.

\section{Conclusion}

Using event-related fMRI together with brief laser pain stimuli of different intensities, we were able to precisely characterize regions within the ACC according to their individual SRF. With respect to pain-related processing, we confirmed previous studies showing pain-related regions in the perigenual ACC and the ventral pACC. Distinct from these pain-related regions, activations in the dorsal aACC were found to code stimulus perception without additional discrimination of pain or stimulus intensities. This response pattern is unrelated to pain processing per se but seems to be associated with working memory or attention to pain. Most interestingly, we discovered an area in the dorsal pACC that showed a BOLD signal that was indicative of basic sensory processing instead of affective pain processing.

Response patterns in the ACC mirror those found in other cortical areas: SI showed a response profile indicating basic sensory processing, SII and the anterior insula showed pain-related responses, and the dorsolateral prefrontal and parietal cortex showed a binary, stimulus perception-related response pattern. Thus, our data support the notion that through projections from all of those regions the ACC can integrate a wide range of pain-relevant information and generate an adequate response through its motor areas situated immediately posterior to the laser-stimulus-related responses.

\section{REFERENCES}

Bouckoms AF (1989) Psychosurgery for pain. In: Textbook of pain (Wall PD, Melzack R, eds), pp 868-881. Edinburgh: Churchill Livingstone. Bromm B, Lorenz J (1998) Neurophysiological evaluation of pain. Electroencephalogr Clin Neurophysiol 107:227-253.

Bromm B, Treede RD (1991) Laser-evoked cerebral potentials in the assessment of cutaneous pain sensitivity in normal subjects and patients. Rev Neurol 147:625-643.

Büchel C, Dolan RJ (2000) Classical fear conditioning in functional neuroimaging. Curr Opin Neurobiol 10:219-223.

Büchel C, Holmes AP, Rees G, Friston KJ (1998) Characterizing stimulus-response functions using nonlinear regressors in parametric fMRI experiments. NeuroImage 8:140-148.

Bushnell MC, Duncan GH (1989) Sensory and affective aspects of pain perception: is medial thalamus restricted to emotional issues? Exp Brain Res 78:415-418.

Casey KL (2000) Concepts of pain mechanisms: the contribution of functional imaging of the human brain. Prog Brain Res 129:277-287.

Casey KL, Minoshima S, Berger KL, Koeppe RA, Morrow TJ, Frey KA (1994) Positron emission tomographic analysis of cerebral structures activated specifically by repetitive noxious heat stimuli. J Neurophysiol $71: 802-807$

Casey KL, Minoshima S, Morrow TJ, Koeppe RA (1996) Comparison of human cerebral activation patterns during cutaneous warmth, heat pain, and deep cold pain. J Neurophysiol 76:571-581.

Coghill RC, Sang CN, Maisog JM, Iadarola MJ (1999) Pain intensity processing within the human brain: a bilateral, distributed mechanism. J Neurophysiol 82:1934-1943.

Corbetta M, Kincade JM, Ollinger JM, McAvoy MP, Shulman GL (2000) Voluntary orienting is dissociated from target detection in human posterior parietal cortex. Nat Neurosci 3:292-297.

Craig AD, Reiman EM, Evans A, Bushnell MC (1996) Functional imaging of an illusion of pain. Nature 384:258-260.

Davis KD, Hutchison WD, Lozano AM, Dostrovsky JO (1994) Altered pain and temperature perception following cingulotomy and capsulotomy in a patient with schizoaffective disorder. Pain 59:189-199.

Davis KD, Taylor SJ, Crawley AP, Wood ML, Mikulis DJ (1997) Functional MRI of pain- and attention-related activations in the human cingulate cortex. J Neurophysiol 77:3370-3380.

Davis KD, Hutchison WD, Lozano AM, Tasker RR, Dostrovsky JO (2000) Human anterior cingulate cortex neurons modulated by attention-demanding tasks. J Neurophysiol 83:3575-3577.

Devinsky O, Morrell MJ, Vogt BA (1995) Contributions of anterior cingulate cortex to behaviour. Brain 118:279-306.

Dum RP, Strick PL (1996) Spinal cord terminations of the medial wall motor areas in macaque monkeys. J Neurosci 16:6513-6525.

Evans AC, Collins DL, Mills SR, Brown ED, Kelly RL, Peters TM (1993) 3D statistical neuroanatomical models from 305 MRI volumes. Proceedings of the Institute of Electrical and Electronics Engineering Nuclear Science Symposium and Medical Imaging 3:1813-1817. 
Fink GR, Frackowiak RS, Pietrzyk U, Passingham RE (1997) Multiple nonprimary motor areas in the human cortex. J Neurophysiol 77:2164-2174.

Foltz EL, White LE (1968) The role of rostral cingulotomy in pain relief. Int J Neurol 6:353-373.

Friston KJ, Ashburner J, Frith CD, Poline J-B, Heather JD, Frackowiak RSJ (1995a) Spatial registration and normalization of images. Hum Brain Mapp 2:1-25.

Friston KJ, Holmes AP, Poline J-B, Grasby PJ, Williams SCR, Frackowiak RSJ, Turner R (1995b) Analysis of fMRI time-series revisited. NeuroImage 2:45-53.

Friston KJ, Holmes AP, Worsley KP, Poline J-B, Frith CD, Frackowiak RSJ (1995c) Statistical parametric maps in functional imaging: a general linear approach. Hum Brain Mapp 2:189-210.

Friston KJ, Holmes AP, Price CJ, Buchel C, Worsley KJ (1999) Multisubject fMRI studies and conjunction analyses. NeuroImage 10:385-396.

Gitelman DR, Nobre AC, Parrish TB, Labar KS, Kim YH, Meyer JR, Mesulam MM (1999) A large-scale distributed network for covert spatial attention. Further anatomical delineation based on stringent behavioural and cognitive controls. Brain 122:1093-1106.

Hutchison WD, Davis KD, Lozano AM, Tasker RR, Dostrovsky JO (1999) Pain-related neurons in the human cingulate cortex. Nat Neurosci 2:403-405.

Jones AKP, Brown WD, Friston KJ, Qi LY, Frackowiak RSJ (1991) Cortical and subcortical localization of response to pain in man using positron emission tomography. Proc R Soc Lond B Biol Sci 244:39-44.

Kwan CL, Crawley AP, Mikulis DJ, Davis KD (2000) An fMRI study of the anterior cingulate cortex and surrounding medial wall activations evoked by noxious cutaneous heat and cold stimuli. Pain 85:359-374.

LaBar KS, Gatenby JC, Gore JC, LeDoux JE, Phelps EA (1998) Human amygdala activation during conditioned fear acquisition and extinction: a mixed-trial fMRI study. Neuron 20:937-945.

Petit L, Courtney SM, Ungerleider LG, Haxby JV (1998) Sustained activity in the medial wall during working memory delays. J Neurosci 18:9429-9437.

Peyron R, Garcia-Larrea L, Gregoire MC, Costes N, Convers P, Lavenne F, Mauguiere F, Michel D, Laurent B (1999) Haemodynamic brain responses to acute pain in humans: sensory and attentional networks. Brain 122:1765-1780.

Peyron R, Laurent B, Garcia-Larrea L (2000) Functional imaging of brain responses to pain. A review and meta-analysis (2000). Neurophysiol Clin 30:263-288.

Picard N, Strick PL (1996) Motor areas of the medial wall: a review of their location and functional activation. Cereb Cortex 6:342-353.

Ploghaus A, Tracey I, Gati JS, Clare S, Menon RS, Matthews PM,
Rawlins JN (1999) Dissociating pain from its anticipation in the human brain. Science 284:1979-1981.

Ploner M, Schmitz F, Freund HJ, Schnitzler A (2000) Differential organization of touch and pain in human primary somatosensory cortex. J Neurophysiol 83:1770-1776.

Porro CA, Cettolo V, Francescato MP, Baraldi P (1998) Temporal and intensity coding of pain in human cortex. J Neurophysiol 80:3312-3320.

Rainville P, Duncan GH, Price DD, Carrier B, Bushnell MC (1997) Pain affect encoded in human anterior cingulate but not somatosensory cortex. Science 277:968-971.

Sawamoto N, Honda M, Okada T, Hanakawa T, Kanda M, Fukuyama H, Konishi J, Shibasaki H (2000) Expectation of pain enhances responses to nonpainful somatosensory stimulation in the anterior cingulate cortex and parietal operculum/posterior insula: an event-related functional magnetic resonance imaging study. J Neurosci 20:7438-7445.

Schouenborg J, Holmberg H, Weng HR (1992) Functional organization of the nociceptive withdrawal reflexes. II. Changes of excitability and receptive fields after spinalization in the rat. Exp Brain Res 90:469-478.

Sikes RW, Vogt BA (1992) Nociceptive neurons in area 24 of rabbit cingulate cortex. J Neurophysiol 68:1720-1732.

Spiegel J, Hansen C, Treede R (2000) Clinical evaluation criteria for the assessment of impaired pain sensitivity by thulium-laser evoked potentials. Clin Neurophysiol 111:725-735.

Talbot JD, Marrett S, Evans AC, Meyer E, Bushnell MC, Duncan GH (1991) Multiple representations of pain in human cerebral cortex. Science 251:1355-1358.

Talbot JD, Villemure JG, Bushnell MC, Duncan GH (1995) Evaluation of pain perception after anterior capsulotomy: a case report. Somatosens Mot Res 12:115-126.

Timmermann L, Ploner M, Haucke K, Schmitz F, Baltissen R, Schnitzler A (2001) Differential coding of pain intensity in the human primary and secondary somatosensory cortex. J Neurophysiol 86:1499-1503.

Tölle TR, Kaufmann T, Siessmeier T, Lautenbacher S, Berthele A, Munz F, Zieglgansberger W, Willoch F, Schwaiger M, Conrad B, Bartenstein P (1999) Region-specific encoding of sensory and affective components of pain in the human brain: a positron emission tomography correlation analysis. Ann Neurol 45:40-47.

Vogt BA, Pandya DN (1987) Cingulate cortex of the rhesus monkey. II. Cortical afferents. J Comp Neurol 262:271-289.

Vogt BA, Pandya DN, Rosene DL (1987) Cingulate cortex of the rhesus monkey. I. Cytoarchitecture and thalamic afferents. J Comp Neurol 262:256-270.

Worsley KJ (1994) Local maxima and the expected Euler characteristic of excursion sets of $\chi^{2}, \mathrm{~F}$, and t fields. Adv Appl Prob 26:13-42.

Worsley KJ, Friston KJ (1995) Analysis of fMRI time-series revisited again. NeuroImage 2:173-181. 